\title{
Endoscopic Endonasal Reconstructive Methods to the Anterior Skull Base
}

\author{
Srikant Chakravarthi, MD, MSc ${ }^{1}$ Lior Gonen, MD ${ }^{1}$ \\ Sammy Khalili, MD, MSc ${ }^{2}$ Amin Kassam, MD ${ }^{1}$
${ }^{1}$ Department of Neurosurgery, Aurora Neuroscience Innovation Institute, Aurora St. Luke's Medical Center, Milwaukee, Wisconsin
2 Department of Otorhinolaryngology, Aurora Neuroscience Innovation Institute, Aurora St. Luke's Medical Center, Milwaukee, Wisconsin

Alejandro Monroy-Sosa, MD

\begin{abstract}
Address for correspondence Amin Kassam, MD, Aurora Neuroscience Innovation Institute, Aurora St. Luke's Medical Center, 2801 West Kinnickinnic River Parkway, Suite 630, Milwaukee, WI 53215 (e-mail: amin.kassam@aurora.org).
\end{abstract}

\author{
Abstract \\ Keywords \\ - expanded endoscopic \\ endonasal approach \\ - cerebrospinal fluid \\ - nasoseptal flap \\ - middle turbinate flap \\ - inferior turbinate flap \\ - pericranial flap \\ - temporoparietal \\ fascial flap
}

The success of expanded endoscopic endonasal approaches (EEAs) to the anterior skull base, sellar, and parasellar regions has been greatly aided by the advancement in reconstructive techniques. In particular, the pedicled vascularized flaps have been developed and effectively cover skull base defects of varying sizes with a significant reduction in postoperative CSF leaks. There are two aims to this review: (1) We will provide our current, simplified reconstruction algorithm. (2) We will describe, in detail, the relevant anatomy, indications/contraindications, and surgical technique, with a particular emphasis on the nasoseptal flap (NSF). The inferior turbinate flap (ITF), middle turbinate flap (MTF), pericranial flap (PCF), and temporoparietal fascial flap (TPFF) will also be described. The NSF should be the primary option for reconstruction of majority of skull base defects following endonasal endoscopic surgery. In general, for the planum, cribriform, and upper two-thirds of the clivus, the NSF is ideal. For the lower-third of the clivus, the NSF may not be adequate and may require additional reconstructive options. Although limited in reach or more technically challenging, these reconstructive flaps should still be considered and kept in the surgical algorithm.
Endoscopic approaches and techniques to the anterior skull base, sellar, and parasellar regions have contributed significantly to the field of skull base surgery, facilitating the resection of complex skull base lesions with a minimal surgical footprint. The most clinically significant complication with these approaches has been the incidence of postoperative cerebrospinal fluid (CSF) leak. ${ }^{1}$ Once the initial feasibility and efficacy were established, demonstrating improved neurological outcomes, reconstruction, CSF leaks became the primary challenge. The expanded endoscopic endonasal approach (EEA) followed the same progression as open skull base surgery such that reconstruction from grafts to vascularized flaps allowed a concomitant reduction in CSF leak rates, which are now in the range of 1 to $5 \% .^{2-4}$

The EEAs have been organized into the following corridors along the coronal and sagittal planes, which span the entire ventral anterior, middle, and posterior cranial fossa, providing access via a series of modular approaches; specifically, the transfrontal, transcribriform, transplanum-transtuberculum, transsellar, transclival, transodontoid, and respective coronal modules. ${ }^{5}$ The reconstruction is primarily dependent on the anatomic region and module undertaken relative to the availability of local and regional vascularized pedicled flaps. Several attempts at categorizing reconstruction options have been described, the most notable is by Patel et al. ${ }^{6}$

There exists significant literature on the use of grafts, and as stated, as the EEA approaches evolved, so has the reconstruction from grafts to flaps. To avoid reiteration, in this article, we outline a pragmatic algorithmic approach focused exclusively on vascularized flap reconstruction; explicitly, we share with the readers our specific reconstruction algorithm that we have been using over the past decade to
Issue Theme Skull Base Reconstruction; Guest Editor: Yadranko Ducic, MD
Copyright (c) 2017 by Thieme Medical Publishers, Inc., 333 Seventh Avenue, New York, NY 10001, USA. Tel: +1(212) 584-4662.
DOI https://doi.org/ 10.1055/s-0037-1607274. ISSN 1535-2188. 
reconstruct defects following EEA. In general, flaps can be considered in three broad categories:

1. Local:

a. Middle turbinate flap (MTF)

b. Inferior turbinate flap (ITF)

c. Nasoseptal flap (NSF)

d. Lateral wall flap (LWF)

2. Regional:

a. Midline: pericranial flap (PCF)

b. Paramedian: temporoparietal fascial flap (TPFF)

3. Distant (free flaps):

a. Anterior lateral thigh (ALT) flap

b. Radial forearm flap (RFF)

While we have used all of the above, for the purposes of this article and in the interest of providing a pragmatic algorithm, we will focus on the local flaps (excluding the lateral wall flap, as we consider this to be an advanced reconstruction approach) and the common regional flaps.

\section{Endoscopic Skull Base Reconstruction Algorithm}

The first critical issue prior to deciding which reconstructive method to use is confirming the presence or absence of a CSF leak. Often, when there is no CSF leak present, no reconstruction is required. Next, the operator must decide if the leak is high flow or low flow, which in turn, determines the need for temporary CSF diversion, usually in the form of a lumbar drain.

Explicitly, a high-flow CSF leak, which by definition occurs following a wide opening of two arachnoid cisterns and a ventricular cavity (third ventricle), leads to a modified algo- rithm, i.e., under all circumstances, a vascularized pedicled flap is selected if possible and augmented with onlay (over the flap) fat followed by a lumbar drain.

-Fig. 1 describes the reconstructive options available. For local defects along the sagittal plane, we prefer the nasoseptal flap (NSF) for the cribriform, planum, and upper twothirds of the clivus. For the lower-third of the clivus, the NSF may not be adequate, and other reconstructive options may then be considered. In general, the NSF is preferred over the lateral wall flap (LWF) and before any other regional flaps.

Multilayered reconstruction in a radial fashion from inside out is preferred, i.e., every layer opened must be categorically reconstructed. Specifically, reconstruction of the arachnoid consists of an inlay collagen implant; note we generally avoid intradural fat, as it can create mass effect and is not a reliable long-term construct. Next, the dura now needs to be reconstructed using the appropriate local, or if unavailable, regional pedicled vascularized flap. In the case of high-flow leaks, as discussed, this can be augmented with a subsequent onlay of fat that is essentially used as a biological dressing in combination with temporary CSF diversion. For local flaps, in general, the NSF is preferred over the LWF due to technical complexities in raising the latter.

For small defects, based on anatomic region, MTF (cribriform and planum) and ITF (upper-third of the clivus) can be considered, though these have very limited range. With respect to regional flaps, for midline defects, PCF is preferred. For paramedian defects, the TPFF is preferred (-Fig. 2).

However, independent of the specific flap, and unlike conventional open skull base surgery, the challenge with EEA is the need to not only consider but actually raise the flap prior to completion of the approach. Unlike most reconstructions, many endonasal approaches involve completing the reconstructive options prior to initiating actual resection. This is an important consideration when choosing reconstructive

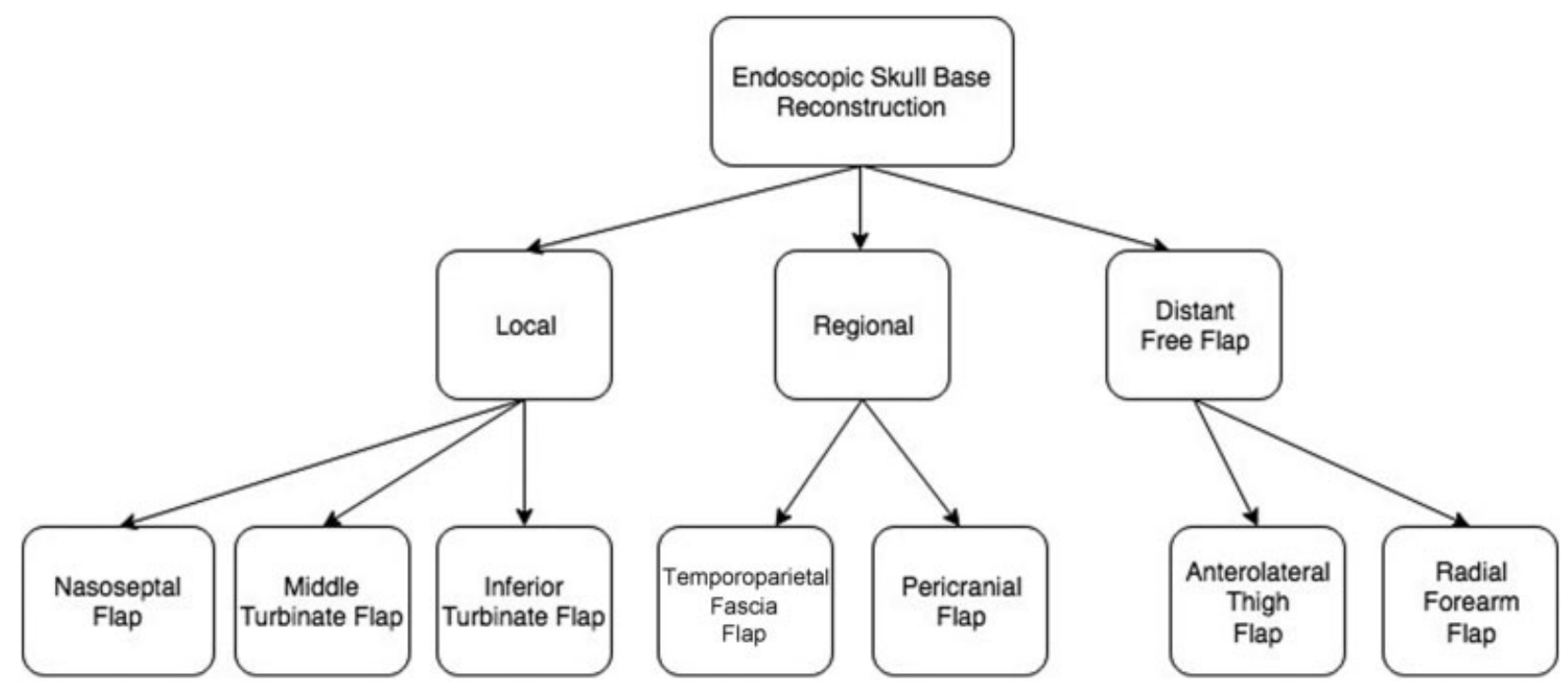

Fig. 1 Endoscopic skull base reconstruction algorithm. This schematic provides a general overview of possible reconstructive options following EEA to the skull base. There are three main categories: local, regional, and distant free flaps. The most common local flaps are the NSF, LWF, MTF, and ITF. The NSF should be considered the primary reconstructive flap. The main regional flaps are the transfrontal PCF and the TPFF. Distant free flap options are the ALT and RFF. ALT, anterolateral thigh; EEA, endoscopic endonasal approach; ITF, inferior turbinate flap; LWF, lateral wall flap; MTF, middle turbinate flap; NSF, nasoseptal flap; PCF, pericranial flap; RFF, radial forearm flap, TPFF, temporoparietal fascial flap. 


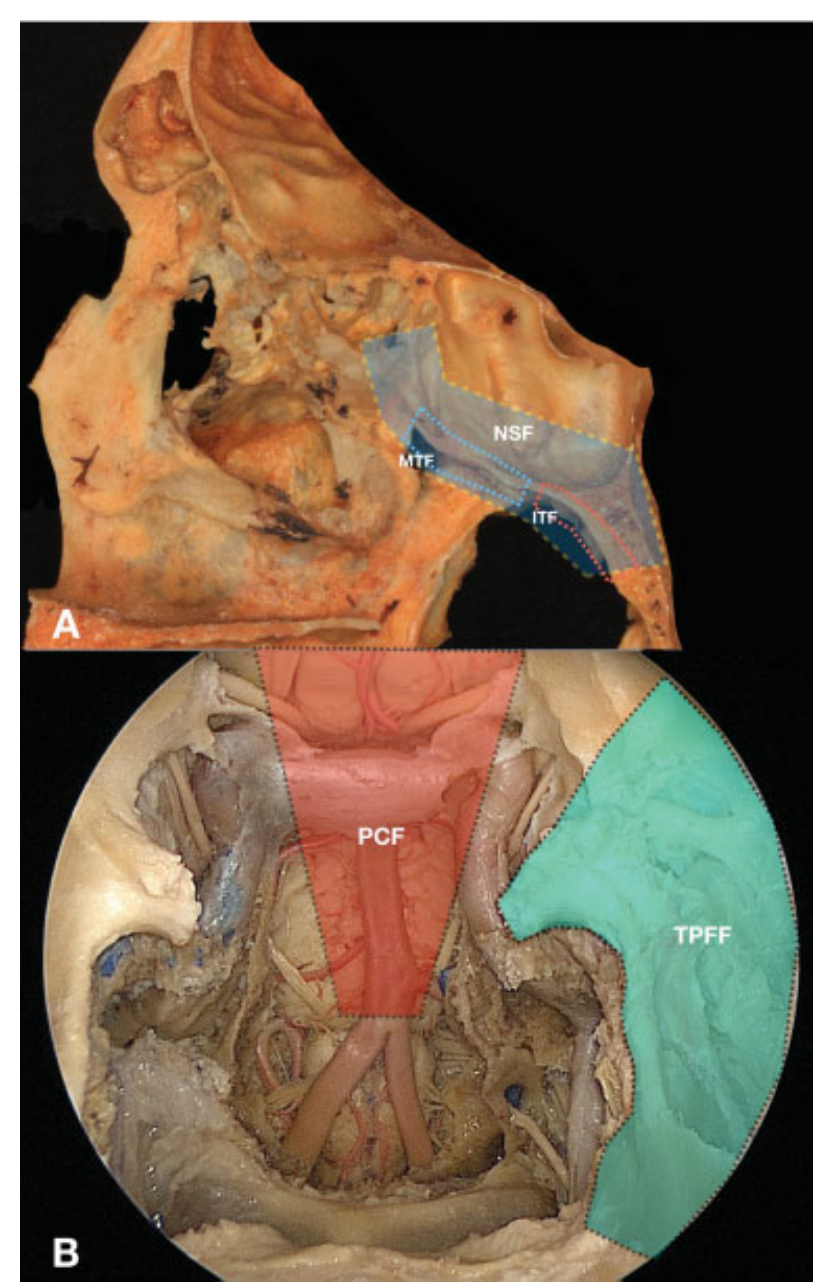

Fig. 2 Anatomic coverage of reconstructive flaps. (A) Sagittal plane depicting the approximate coverage of the NSF, MTF, and ITF. The NSF can adequately cover the cribriform, planum, and upper two-thirds of the clivus. The MTF and ITF can cover small defects of the cribriform and planum and upper one-third of the clivus, respectively. (B) The coverage of the PCF is the coronal midline plane. For paramedian defects, the TPFF is preferred. ITF, inferior turbinate flap; MTF, middle turbinate flap; NSF, nasoseptal flap; PCF, pericranial flap; TPFF, temporoparietal fascial flap.

options for various skull base defects and planning the size (-Fig. 2).

\section{Technical Nuances}

\section{Nasoseptal Flap}

Much of the acceptance and standardization of EEAs to ventral skull base, sellar, and parasellar regions can be attributed to the development of the pedicled NSF. ${ }^{7}$ Prior to this, nonvascularized tissue grafts and synthetic materials were the mainstay constructs for reconstruction of the defect between the subarachnoid space and the sinonasal tract, which was considered to be one of the greatest obstacles to an effective EEA surgery and reduction of patient morbidity and mortality. In particular, the primary factors in morbidity and mortality were postoperative CSF leaks, which according to Harvey et $\mathrm{al}^{8}$ were 15.6 and $6.7 \%$ for free grafts and vascularized reconstruc- tion, respectively. Therefore, the NSF has been the mainstay for reconstruction of large skull base dural defects (- Fig. 3). ${ }^{8}$

\section{Nasal Surgical Landmarks}

The NSF is a vascular pedicle flap that is supplied by the posterior nasoseptal artery, a branch of sphenopalatine artery. The pterygomaxillary segment of the internal maxillary artery (IMAX) travels from the pterygomaxillary fissure through the pterygopalatine fossa and to the nasal cavity via the sphenopalatine foramen (SPF). The SPF is formed between the orbital process and the sphenoid process of the palatine bone. The anterolateral segment of the SPF is surrounded by the crista ethmoidalis as an oblique bony ridge, and thereby serves as the key landmark for the identification of the SPF. In addition, this must be removed to uncover the terminal IMAX.

\section{Surgical Technique}

The NSF is currently considered the mainstay reconstructive method for all dural defects along the sagittal plane above the lower-third of the clivus, following an EEA. In almost all cases, we elevate the NSF on the right (contralateral) side as long as the septal mucosa is intact. The indications for a leftsided NSF include (1) a right septal neoplastic process and (2) a right-sided transpterygoid approach that would threaten the pedicle, necessitating a contralateral left-sided flap.

Our working concept for all EEAs procedures is creation of a "cavity-and-a-half" for adequate visualization. The half cavity allows for freedom and mobility of the endoscope, and the full sinus cavity serves as a working cavity for the bimanual, binasal dissection. Preparation of the NSF generally requires the following stages: (1) ipsilateral inferior turbinate lateralization, (2) ipsilateral middle turbinate (lower one-third) resection, (3) contralateral outfracture of the middle turbinate, (4) elevation of the NSF, (5) posterior septectomy, (6) wide bilateral sphenoidotomies, and (7) posterior and ethmoidectomies (optional).

The first step is to outfracture and lateralize the ipsilateral inferior turbinate. This can be accomplished with either a Goldman freer or Cottle elevator, often in conjunction with nasal decongestion with either oxy- or xylometazoline. The middle turbinate is often infractured after the inferior turbinate is outfractured, as it is first prepared with an injection of $1 \%$ lidocaine and epinephrine and allowed to set for approximately 3 minutes. With either endoscopic scissors or a Colorado Bovie needle tip monopolar cautery, the lowerthird of the middle turbinate is cut and pulled downward, leaving a residual stump. Hemostasis of the middle turbinate branch of the sphenopalatine artery is then achieved with either an endoscopic bipolar or suction cautery. Care is taken not to compromise the pedicle of the NSF, as this can easily occur. We do recommend consideration of posterior ethmoidectomies bilaterally for two main reasons-first, it allows for a wider approach and exposure for tumor removal; second, it allows for greater mobility and dexterity in harvesting the flap and mobilizing the pedicle as laterally as possible. Apart from creating a wide endonasal working corridor by completing a posterior ethmoidectomy, the resection of the 


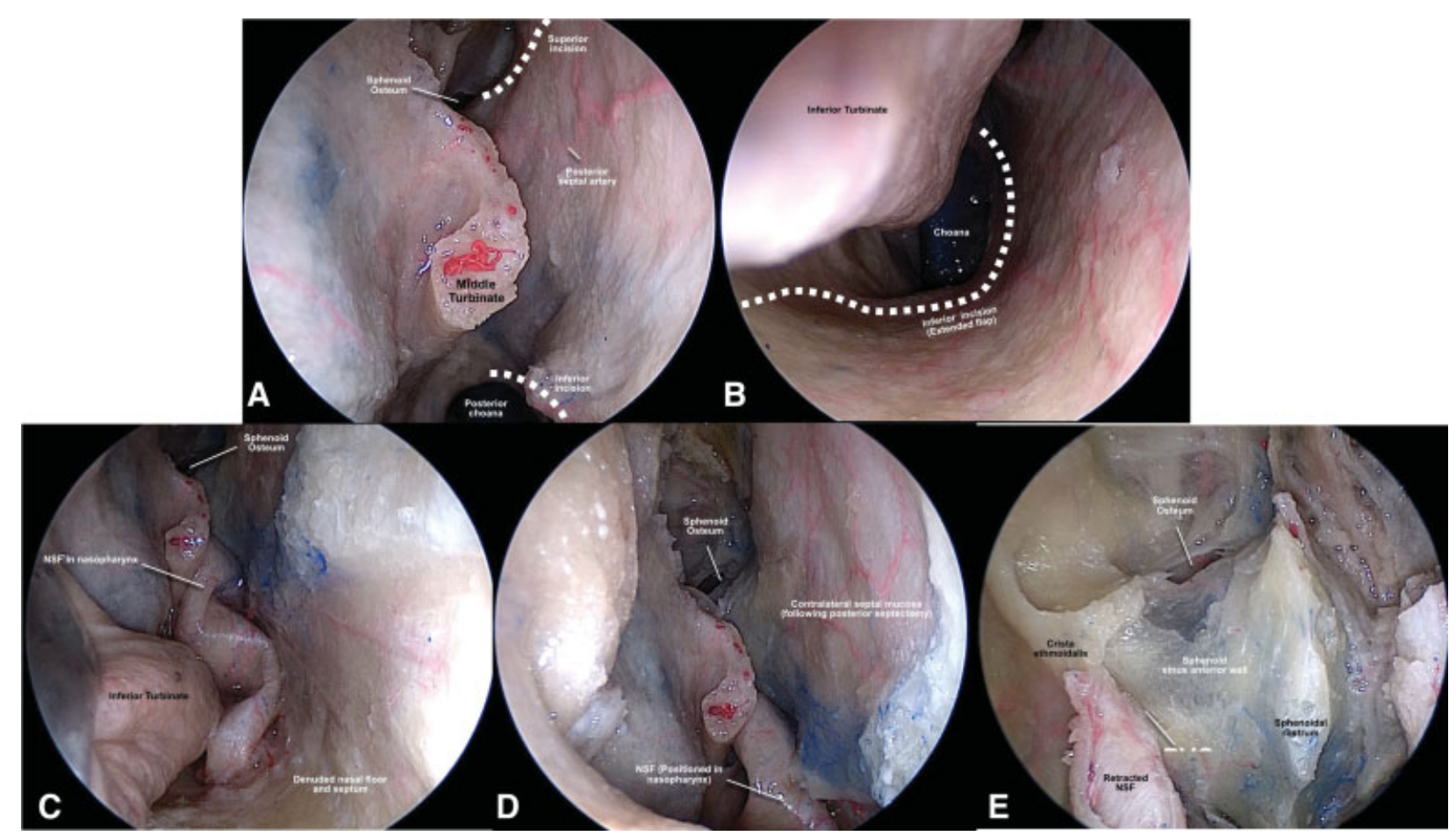

Fig. 3 Pedicled NSF. (A) Lateralizing and outfracturing the middle turbinate provide an unhindered view of the nasal cavity. Then, superior and inferior incisions are made. The superior incision is made at the level of the sphenoid ostium, and the inferior incision is made at the level of the posterior choana. (B) As part of an extended flap, a long inferior incision is made at the level of the posterior choana. (C) After NSF is lifted, it is pushed into the nasopharynx, with a resulting denuded nasal septum and floor. (D) Contralateral septal mucosa following posterior septectomy. (E) Retracted NSF in relation to the crista ethmoidalis, sphenoid ostium, and anterior wall of sphenoid sinus. NSF, nasoseptal flap.

lower third of the ipsilateral middle turbinate facilitates mobilization of the NSF vascular pedicle, allowing it to be stored out of way during the remainder of the approach and resection. On the contralateral nasal cavity, the middle turbinate is outfractured and lateralized. This maneuver will facilitate the binasal/bimanual approach to the sphenoid sinus and allow for a greater surface area to raise the NSF.

The size and shape of the flap should be tailored according to the anticipated size of the defect and often requires a certain degree of oversizing to accommodate for subsequent consolidation and contraction. The flap is created by making two parallel incisions from a posterior to anterior direction, with a vertical incision joining the two, and anterior to the inferior turbinate. The raise is always initiated by performing the inferior cut first (made with a Bovie fitted with an arthroscopic needle tip) to avoid dripping of blood from above as a result of gravity. The inferior incision is made along the inferior aspect of the nasal choana on the nasal floor; the most common mistake for the uninitiated is to make this cut too high and in the face of the sphenoid floor. There are three pedicles to the flap and if the cut is too high, the lower pedicle is transected. To avoid this, the lower cut should begin just medial to the torus tubarius and then continue following the free edge of the posterior nasal septum. This incision ends just posteriorly below the floor of the sphenoid sinus. An extended, wider flap can be made by making an inferior cut lateral and inferior to the inferior turbinate.

The superior incision is initiated at the level of the sphenoid ostium and is followed anteriorly inferior to the olfactory epithelium (which is often thicker-typically double the thickness and more yellow in appearance than normal nasal mucosa), along the septum and extending it anteriorly along the limen nasi. A good general rule is to stay under the vertical height of the middle turbinate to preserve olfaction, once past the anterior limit of the middle turbinate, the cut is carried superiorly; therefore, the flap is Y-shaped and not rectangular. In addition, in an effort to spare the nasal mucosa, our priority is to spare the mucosa along the nasal roof to decrease the risk of olfactory nerve dysfunction. An anterior vertical incision at the level of the mucocutaneous junction at the anterior portion of the nares is then made to connect the two.

Using a suction Cottle elevator, the flap is then elevated along the subperichondrial plane in an anterior to posterior direction. This will also allow the surgeon to check if all the incisions were made through the periosteum and perichondrium. We recommend elevating the incised mucosal edges by 1 to $2 \mathrm{~mm}$ to make sure these are of adequate depth. The flap is elevated up to the anterior face of the sphenoid sinus with the vascular pedicle contained within the strip of mucosa between the natural ostium of the sphenoid sinus and the posterior choana. After the NSF is elevated, it can be stored in the nasopharynx or alternatively, in a maxillary antrostomy that can be created for storage (the other advantage of creating a maxillary antrotomy is that it also creates another point of egress of blood). One important modification at this juncture is the lateral mobilization of the NSF with identification of the SPF. We remove the uncinate process to expose the maxillary sinus ostium and then create a wide nasoantral window 
through the natural sinus ostia, thus decreasing the likelihood of creating recirculation syndrome later. The SPF is then exposed following elevation of the mucoperiosteum of the lateral nasal wall, located just posterior to newly created nasoantral window. In addition, the crista ethmoidalis is removed with a Kerrison rongeur, and subsequently, the posterior nasal and sphenopalatine arteries are identified and mobilized out of the SPF. This step, in conjunction with a posterior ethmoidectomy, will greatly aid in the rotational movement of the flap. Removal of the crista ethmoidalis has two advantages: (1) it enables lateral mobilization of the flap and facilitates wide exposure of the floor of the sphenoid sinus, and (2) it facilitates the full length of the flap by bringing the flap to the same level as the anterior skull base. Storage of the NSF is important for adequate visualization of the remaining procedure. A posterior septectomy can then be made to allow bimanual access to the posterior nasal corridor and for eventual placement of the contralaterally based flap to extend to the ipsilateral side.

\section{Alternative Vascularized Intranasal Flaps}

The NSF is considered the workhorse for endoscopic skull base reconstruction. It is highly reliable compared with other flap options and relatively straightforward to raise with relative lower morbidity. However, there are several relative contraindications that need to be considered; most notably, prior septectomy and tumors involving the septum/pedicle. As such, other local vascularized local reconstruction options can be considered, including inferior and middle turbinate flaps. However, it must be noted that given the relative limited reach, these may not solve the reconstruction challenge even if combined, necessitating the need for extranasal regional flaps.

\section{Inferior Turbinate Flap}

\section{Indications}

An inferior turbinate flap (ITF) can be used to cover small clival and sellar defects. It can also be effective as an option of reconstruction, in the face of previous surgery, as in most prior surgeries of the sinonasal cavity, the inferior turbinate is often preserved. Due to the limited length of the flap, it can be combined with middle or contralateral IFT to further fill in the defect (-Fig. 4).

\section{Contraindications}

Contraindications are similar to other considerations, such as tumor invasion, previous surgery, and limited reach.

\section{Surgical Technique}

The vascularization of the ITF is based on the inferior turbinate artery, which is a branch of the posterior lateral nasal artery (a branch of the sphenopalatine artery). The posterior lateral nasal artery first runs along the inferolateral segment of the anterior process of the palatine bone and provides a branch to the middle turbinate medially. As the artery travels anteriorly, it becomes larger, connecting with the angular artery and supplying the anterior blood supply to the inferior turbinate..$^{9-11}$ It is important to consider that certain anatomic variations exist in the blood supply of the sphenopalatine artery, one of which is that the posterior lateral nasal artery may extend anteriorly to the posterior wall of the maxillary antrum. ${ }^{12,13}$ This variant in particular is important to recognize in avoiding injury to the vascular pedicle when performing the maxillary antrostomy and mucoperiosteal elevation.

The first step of ITF harvest is medialization of the inferior turbinate to allow for visualization of the medial surface of the inferior turbinate. To visualize the natural ostium of the maxillary sinus, the uncinate process and the bulla ethmoidalis are then removed. Next, the maxillary sinus ostium should be widened toward the posterior maxillary antrum. With either a Cottle or Freer elevator, the submucoperiosteal mucosa overlying the anterior aspect of the ascending process of the palatine bone is lifted in a posterior direction toward the crista ethmoidalis, sphenopalatine artery, and foramen. Next, in a posterior to anterior direction, two parallel incisions are made to create the superior and inferior margins of the ITF. The superior incision starts at the posterior margin of the middle meatus at the site of the middle meatal antrostomy and extends superiorly to include the posterolateral margin of the nasal wall, along the nasal aperture, with the posterior limit being the Eustachian tube.

The inferior incision is a midline cut along the nasal septum, posteriorly extending to the Eustachian tube. During this step, it is important to sharply dissect the mucoperiosteum around the nasolacrimal duct to prevent its disruption. Next, a vertical incision at the head of the inferior turbinate, at its attachment to the piriform aperture, joins the superior and inferior incisions. Using either a Freer or Cottle elevator, the mucoperiosteum can now be raised from the floor of the inferior turbinate in an anterior to posterior direction and from medial to lateral. At this point, the tedious task of removing the internal cartilage without disrupting the overlying mucosa is now pursued. The pedicled and elevated flap often retains the shape of the inferior turbinate. Much of this is due to the presence of the midline ridge of the inferior turbinate overlying the mucosa. Therefore, the difficult step is to flatten the flap so that it fits securely on the defect. As a result, this bone is removed once the flap is elevated. The flap is then rotated into the skull base defect and set into place. Sometimes, a small Y-shaped cut has to be made in the distal end of the flap to allow for the flap to be placed in a flat position. It is notable that it can be challenging raising the flap after the turbinate bone has been fractured.

\section{Middle Turbinate Flap}

\section{Indications}

The posteriorly-based MTF has limited applications due its relatively small surface when harvested and limited reach. It may be used to reconstruct small defects of the anterior cranial fossa, fovea ethmoidalis, planum sphenoidale, and sellar region. In general, the MTF is technically difficult to elevate due to the thin, overlying mucoperiosteum that is attached to the irregularly shaped middle turbinate bone (-Fig. 5). 

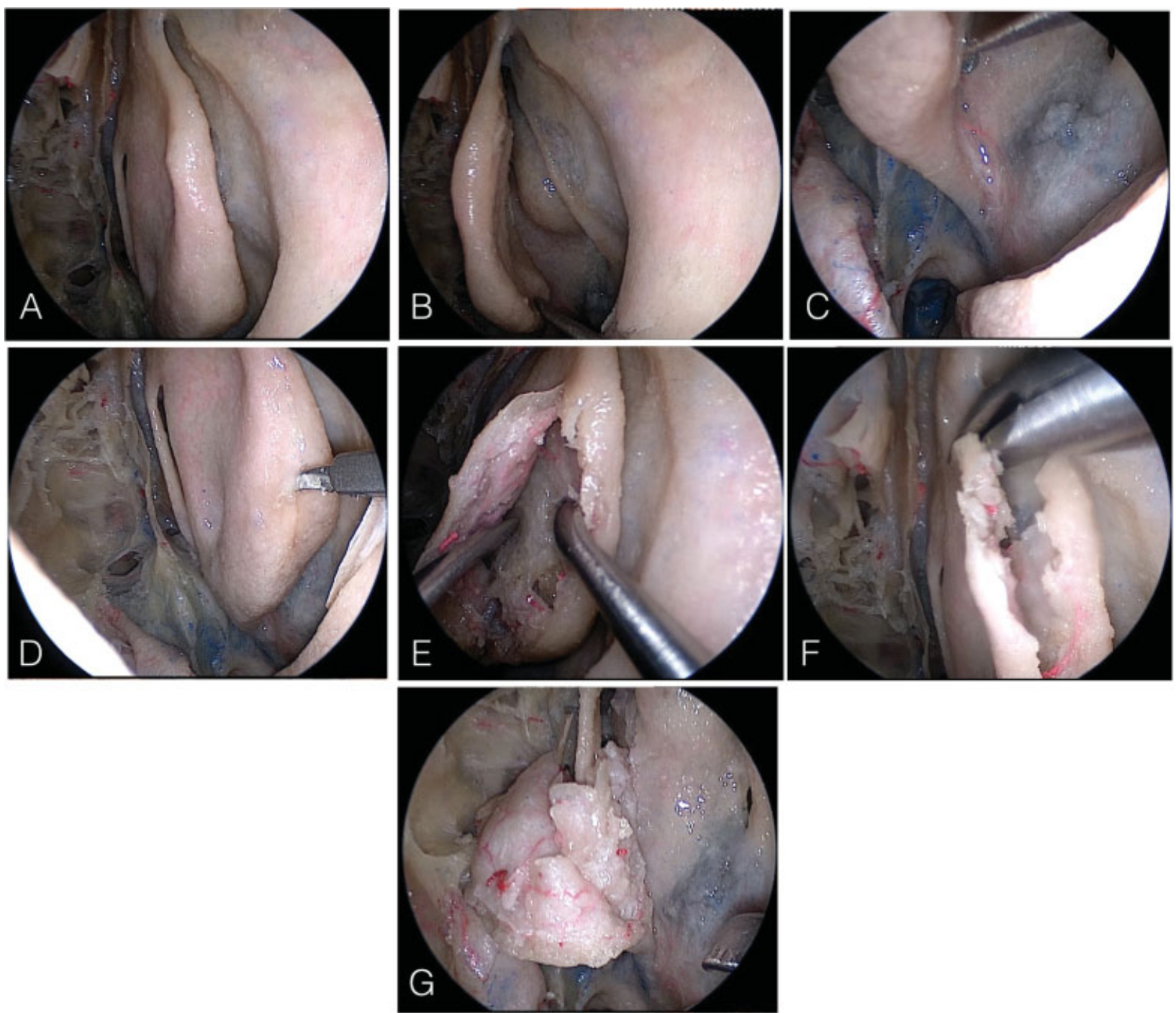

Fig. 4 Middle turbinate flap (MTF). (A) All three turbinates in view. (B). Middle turbinate lateralized. (C) Middle turbinate pedicle (middle turbinate artery, branch of posterior lateral nasal artery). (D) Vertical (superior) incision. (E) Subperiosteal elevation. (F) Horizontal cut. (G) Flap placed on defect site.

\section{Relative Contraindications and Considerations}

We recommend not harvesting an MTF for patients with (1) unstable middle turbinate, (2) anatomic variations, such as concha bullosa, paradoxical middle turbinate, or hypoplasia, and (3) previous surgery of the middle turbinate. There is also a risk of disruption of the bony attachment of the middle turbinate to the cribriform plate, which can result in CSF leak. In addition, as with the ITF, previous sphenopalatine artery ligation is an absolute contraindication.

\section{Surgical Technique}

The MTF receives its vascular supply from the middle turbinate artery, which is a branch of the posterior lateral nasal artery. The branch runs inferior to the middle turbinate and further separates into anterior and posterior segments, which supply the lateral and medial mucosa, respectively.

The first step in harvesting the MTF is by starting with a vertical incision along the anterior margin of the middle turbinate and extending down to the front of the turbinate with the two sides of the concha located on either side of the incision. This can be accomplished with a 15 inch blade or a straight Beaver blade or a Bovie needle tip. The vascular pedicle is along the posterior margins of the flap. The mucoperiosteum can now be elevated in a superior to inferior direction on the medial and lateral surface of the bone. Again, this can be accomplished with either a Freer or Cottle elevator. Anatomically, the posterior attachment of the middle turbinate is the ethmoidal crest and the superior attachment is the vertical lamella. Therefore, when elevating the flap, care must be taken to prevent destabilization or fracturing of the middle turbinate bone, as CSF leak could result. Next, a horizontal cut is made on the lateral surface of the axilla to elevate the lateral aspect of the turbinate. The cut extends posteriorly to the level of the SPF. A similar incision can then be made on the medial surface of middle turbinate. These cuts will allow for full isolation of the posterior pedicle. Dissecting and elevating the flap to the level of the SPF allows for increased length and better arc of rotation. The flap is then rotated and set in place along the defect. 

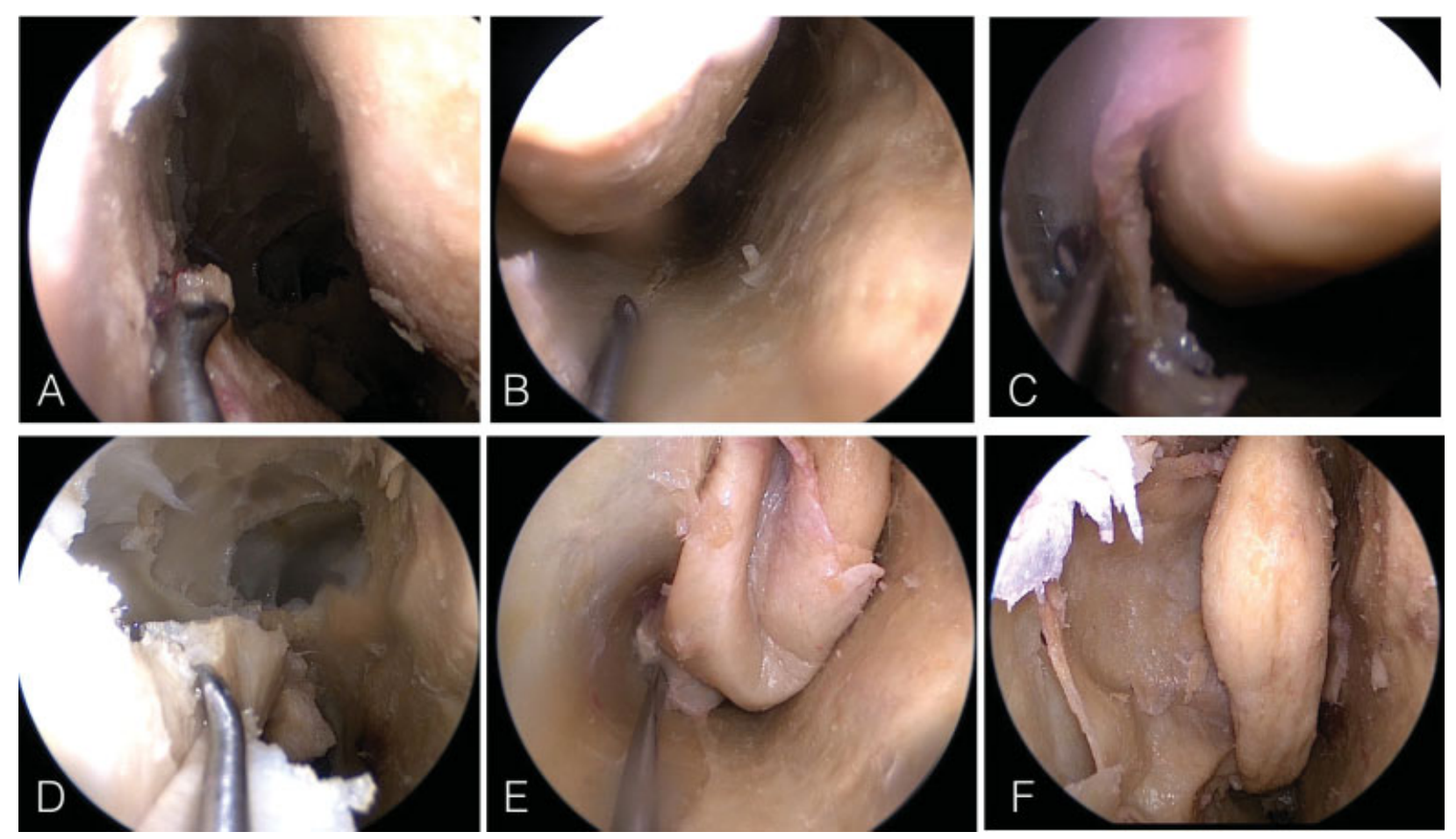

Fig. 5 Inferior turbinate flap (ITF). (A) Superior incision. (B) Inferior incision. (C) Lifting of superior portion of flap. (D) Exposure of flap pedicle. (E) Placement of flap in nasal cavity. (F) Flap in view on defect.

\section{Extranasal ("Regional") Flaps}

\section{Indications}

The extranasal pedicled flaps represent a second-line reconstruction option when the local flaps are not available. The two most common extranasal ("regional") pedicled flaps are the pericranial flap (PCF) and the transpterygoid temporoparietal fascia flap (TPFF). In general, the PCF is easier to raise and tunnel into the nasal cavity, whereas the TPFF is technically more difficult to raise in a tunnel. Therefore, the PCF is preferred over the TPFF. The PCF provides optimal coverage in the midline and middle third of the clivus but can be designed to extend to the inferior third of the clivus all the way to the craniocervical junction. The TPFF provides excellent and reliable coverage for all paramedian plane defects along the coronal plane, as well as very reliable coverage for the entire clivus.

\section{Contraindications}

Contraindications are similar to previous discussion of tumor involvement or unavailability due to previous surgery. An additional contraindication occurs if the respective tunnel is not available.

\section{Transfrontal Pericranial Flap}

\section{Anatomy}

The PCF compromises the ipsilateral pericranium with the pedicle being the ipsilateral supraorbital and supratrochlear neurovascular bundles. The supratrochlear and supraorbital nerves are branches of the ophthalmic division of the tri- geminal nerve and supply the midline forehead to the hairline and forehead and scalp extending back to the vertex, respectively. The supratrochlear and supraorbital arteries are branches of the ophthalmic artery, which arises from the supraclinoid segment of the internal carotid artery as it exits the roof of the cavernous sinus. The supratrochlear and supraorbital notches (or foramina) are the bony elongated prominences that mark the exit of the respective nerves and arteries. The arteries then ascend in the galea frontalis muscular layer providing branches that supply the pericranium. To prevent injury to the neurovascular pedicle, the frontalis muscle should not be separated from the pericranium, when elevating the TPFF (- Fig. 6). ${ }^{14}$

\section{Surgical Steps}

A classical bicoronal incision at the vertex is made and can be extended anteriorly in the preauricular region to enhance the rotation of the flap. Incision along the vertex has two advantages: (1) it prevents transection of the anterior branches of the superficial temporal artery, and (2) it is cosmetically superior. The incision is then carried down to the cranium from one superior temporal line to the other and then to the superficial layer of the deep temporal fascia. We have previously reported on raising the flap through endoscopic approaches, though more recently have gotten away from this, as the bicoronal incision is quite cosmetic. Independent of the skin incision to access the pericranium, it is important to separate the pericranium from the scalp and include it in the flap, as it will lead to a thicker flap.

All flap layers are then elevated to expose the superior orbital rim. It is important at this juncture to locate the relative 

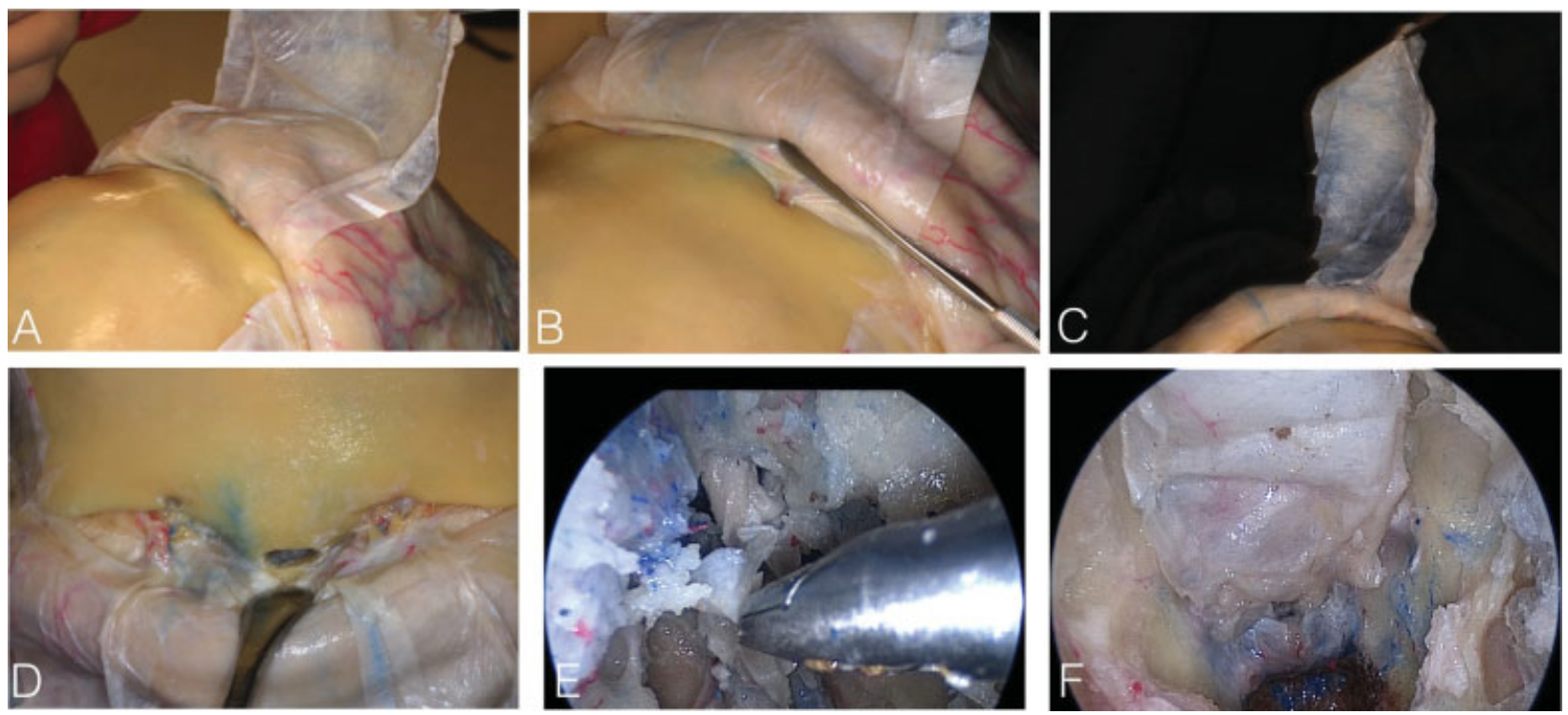

Fig. 6 Transfrontal pericranial flap (PCF). (A) Exposure of periosteal flap layer following a bicoronal incision. (B) View of supraorbital artery emerging from superior orbital rim. (C) Flap is split and this single portion is placed into nasion osteotomy. (D) View of osteotomy at level of nasion, site of insertion of flap. (E) Endonasal view of flap emerging from nasion. (F) Flap in view covering defect.

position of the supraorbital and supratrochlear notch or foramen and be cognizant of the location of the exiting neurovascular bundles $(\sim 10 \mathrm{~mm}$ above the superior orbital rim). According to Patel et al, the goal is for approximately $95 \%$ flap coverage. The tunnel used to deliver the flap is along the glabella; specifically, an osteotomy in the glabella is made and the frontal sinus entered, allowing the flap to be transposed through the nasion osteotomy into the nasal cavity. ${ }^{15}$

There are two important considerations for successful placement of the PCF. First, complete frontal sinus- and ethmoid sinus-ectomies need to be performed to create a tunnel to transfer the flap. Second, if the frontal skull base is sloped and not flattened, it may cause the flap to pull away from the anterior defect. ${ }^{6,15-17}$

\section{Transpterygoid Temporoparietal Fascia Flap}

\section{Anatomy}

The TPFF is another regional vascularized pedicle flap that is anatomically based on a reliable and robust fascial layer overlying the subcutaneous tissue of the temporoparietal region of the scalp. From superficial to deep, the primary soft tissue layers to access the TPFF are as follows: skin, subcutaneous tissue, temporoparietal fascia (TPF), temporal fascia/pericranium, and the temporalis muscle. The TPF is the primary layer that forms the basis of reconstruction in the TPFF. The TPF connects the overlying fibrous septae of the subcutaneous tissue above. The blood supply of the TPF is the superficial temporal artery (STA). After travelling posteriorly to the zygomatic process, the STA terminates at the TPF. According to David and Cheney, ${ }^{18}$ the TPF is 2 to $3 \mathrm{~mm}$ in thickness over the parietal region and extends like a fan from the preauricular region, with a surface area as large as $17 \times 14 \mathrm{~cm} .{ }^{18}$ In addition, the deep temporal fascia consists of dense, white connective tissue layer that overlies the muscle and is continuous with the pericranium above the level of the superior temporal line. As it passes deep to the zygomatic arch, the temporalis muscle forms the lateral boundary of the infratemporal fossa (ITF). This allows communication of the temporal fossa with the ITF ( - Fig. 7 ).

\section{Surgical Technique}

It should be noted that harvesting and transposition of the TPFF is dependent on the extent of the intranasal defect and relative location. We prefer to harvest the flap ipsilateral to the defect or EEA procedure. Prior to incision, it is advised to first locate the superficial temporal artery. An extended hemicoronal incision can then be made. The subdermal and subfollicular planes are dissected to leave the TPF intact. After identifying the parietal and frontal branches of the superficial temporal artery, the margins of the TPFF can then be incised, being cognizant of the plane between the TPFF and the temporalis fascia. The portion of the flap over the skull is incised down through the pericranium. The flap is then elevated to the level of the pedicle. To preserve the frontal branch of the facial nerve, we recommend not extending the interfascial incision past the level of the tragus and lateral eyebrow. If a longer flap is needed, the TPFF can extend to the contralateral temporal fascia incorporating it into the distal portion of the TPFF. This will allow for a much greater surface area for the placement of the eventual TPFF.

Following the elevation of the flap, the tunnel via the intranasal corridor can be developed. The tunnel is initiated by a wide bilateral sphenoidotomy, medial maxillectomy, and exposure of the sphenopalatine canal. If not completed already, an anterior and posterior ethmoidectomy and large maxillary antrostomy should be performed. The SPA and posterior nasal artery are usually identified and clipped at the level of the sphenopalatine foramen. This will allow for an easier dissection of the SPA into the pterygopalatine fossa 

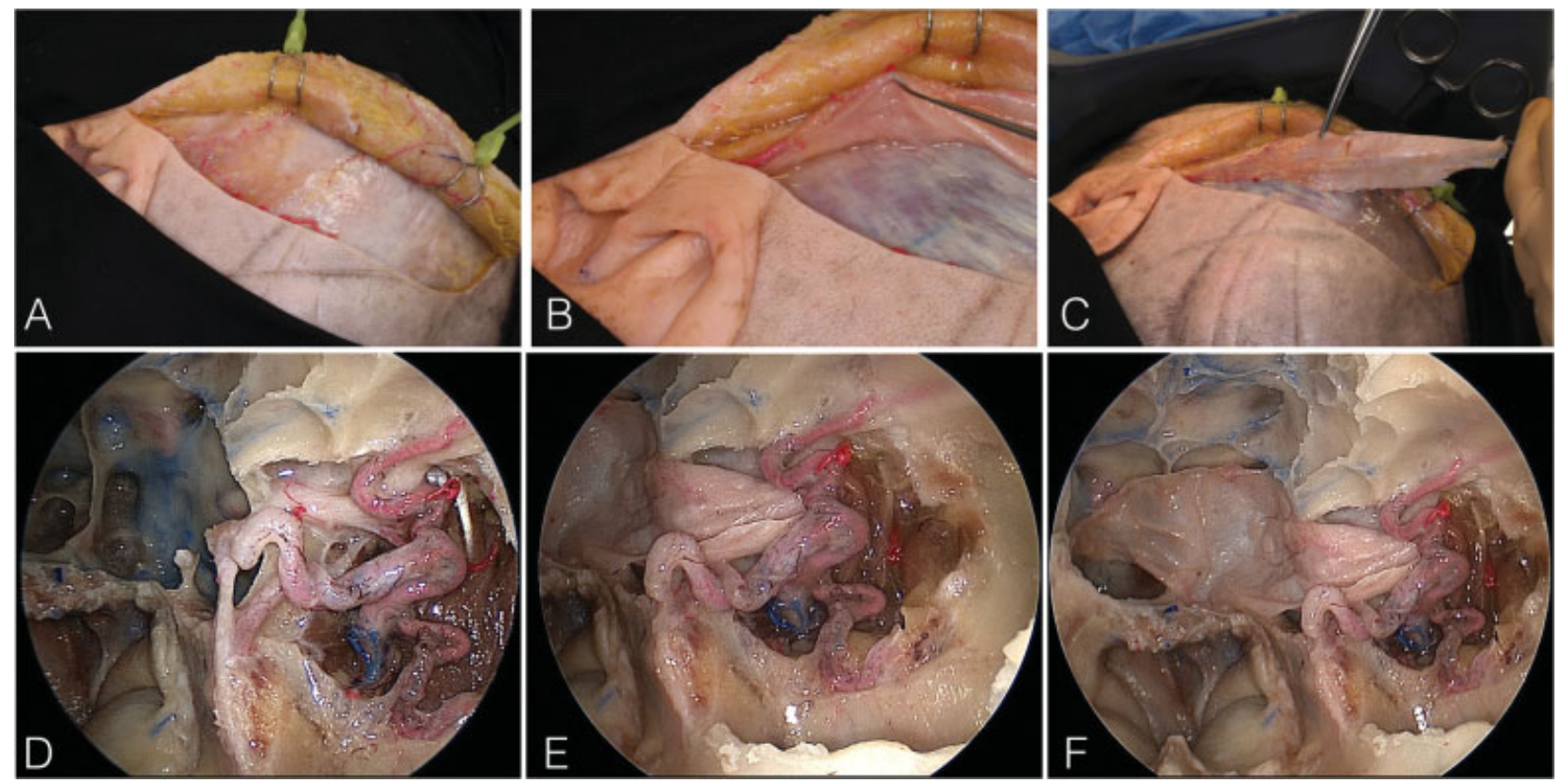

Fig. 7 Temporoparietal fascia flap (TPFF). (A) Hemicoronal incision with view of temporoparietal fascia. (B) View of superior temporal artery. (C) Segment of fascia separated. (D) Endonasal view of pterygopalatine fossa and pterygomaxillary fissure, site of insertion of flap. (E) Flap emerging from pterygomaxillary fissure. (F) Final view of flap covering area of sella and clivus.

(PPF). The key here is to expose the PPF, remove the lateral wall of the maxillary sinus, and open a communication with the ITF. The soft tissue contents of the PPF should now be displaced inferiorly and laterally to expose the pterygoid plates. At this juncture, it is acceptable to sacrifice the vidian nerve, if it cannot be adequately mobilized, but we recommend preserving the pterygopalatine ganglion. Next, the pterygoid plates can be drilled away to give room for the eventual transposition of the TPFF and allow for the distal tunnel to reach the lower clivus if needed.

The next and last step would be to create a wide enough communication between the intranasal and extranasal compartments. A second incision separating the superficial and deep layers of the temporal fascia, 2 to $3 \mathrm{~cm}$ above the zygomatic arch, just at the level of the frontozygomatic suture is made. The primary reason for the superficial and deep separation is to (1) avoid the overlying facial nerve and (2) open an entrance into the deep intranasal corridor along the zygoma to the lateral orbital wall, i.e., this provides access to the region of the lateral pterygoid, lateral maxilla, and TTP.

This dissection can then be extended inferiorly to elevate the periosteum from the medial surface of the zygoma. Next, we can elevate the temporalis muscle to the level of the lateral orbital wall and inferior orbital fissure. This maneuver will create a communication between the temporal, infratemporal, and pterygopalatine fossae. We do not think that a lateral orbital incision is necessary in this case to further separate the temporalis muscle from the zygoma, as the flap can be pulled enough anteriorly for adequate visualization. A guide wire or similar tool can be used to pass the flap through the temporal fossa and then through the infratemporal fossa to enter the pterygomaxillary fissure to enter the pterygopalatine fossae.
Endoscopically, care should be taken to not overly compress the flap, as it may compromise the vascular flow of the flap. ${ }^{19}$

\section{General Considerations}

Often there is a discussion regarding implants, such as plates and bone grafts, "gasket seal"; we have not found this of value and generally avoided this for several reasons:

1. The defect, in our experience, created during the exposure was wide and most often extended to critical structures, such as the carotid artery and optic nerve, precluding wedging implants into the margin.

2. We strongly advocate to avoid putting a foreign body graft between the vascularized flap and the recipient site, as it will impair uptake and vascular reconstruction.

\section{Preparation of the Recipient Site}

There are several key considerations with respect to the recipient site to allow for optimized uptake of the flap:

1. The inset of the flap must be such that it makes contact with bone along its entire length, especially the proximal portion. For the uninitiated, there is a tendency to place only the distal flap directly in contact with the defect, leaving an "air gap" under the proximal flap. The flap will seek bone during the neovascularization process and proximally contract pulling it away from the distal portion where it is meant to cover the defect.

2. All proximal bone must therefore be removed, as any ridge will require the flap to be twice the length of ridge; for example, if the floor of the sphenoid sinus and vomer is $1 \mathrm{~cm}$ vertical, the residual flap will need to climb "uphill and downhill," requiring an additional $2 \mathrm{~cm}$ distance. 
3. There can be no mucosa or foreign body, such as bone wax or fibrin glue, between the flap and the bone to which neovascularization should occur.

4. The arachnoid is separately reconstructed with a simple collagen implant as an inlay graft.

\section{Coverage of the Donor Site}

In an area of significant crusting, the donor site should ideally be covered. In the case of the NSF, we use the contralateral septum to create a reverse vascularized flap.

\section{Discussion}

Endoscopic approaches to the skull base have greatly advanced over the past two decades. The main limitation of the EEA has been the incidence of the CSF leak. However, the development and refinement of reconstructive methods, especially with the vascularized NSF, have greatly reduced the incidence of this complication and as a result improved patient morbidity and mortality. We provided an overview and algorithm of the possible reconstructive methods following an EEA and described in detail, four of the five vascularized flaps that are more commonly used in the clinical setting.

Several studies have described the reconstructive options following an expanded EEA to the skull base. ${ }^{2,5-8,20,21}$ In particular, in collaboration with our colleagues, Hadad et $\mathrm{al}^{22}$ we first introduced the vascularized pedicled NSF that has been the workhorse ever since and the primary reason for a substantial decrease in postoperative CSF leak and infection rates. ${ }^{22}$ Based on our algorithm and experience, the NSF can be successfully placed over the majority of defects, regardless of the size and amount of CSF leak. In addition, the NSF can be tailored or expanded to fit many of the endoscopic corridors, especially in the midline, i.e., transsellar, transplanum, transclival, and transodontoid approaches. ${ }^{23}$ The far paramedian corridors may require transpterygoid approach, which, depending on the location of the lesion, may be too lateral for the NSF, and therefore may require additional methods (i.e., lateral wall or TPFF). As a result of usage of the NSF, our postoperative CSF leak has reduced to less than $1 \%$ from an incidence of 2.2 to $6.6 \%$ in previous reports. ${ }^{3,6}$ This improvement has been greatly aided by preoperative radiological guidance, with computed tomography (CT), magnetic resonance imaging (MRI), and diffusion tensor imaging (DTI), and preplanning of the surgical approach and trajectory, facilitating a more tailored harvest of the NSF.

As mentioned in this review, there are several advantages and limitations of the other reconstructive options. To reiterate, the NSF should be the first consideration in the repair of skull base defects. The LWF is the second most effective flap that we currently use but is often technically challenging, with a higher donor morbidity. The ITF and MTF can be used for small clival and transsphenoidal/transplanum defects, respectively. Due to its small size and location, the ITF is not suitable for anterior cranial fossa or sellar defects, and alternatively, the MTF does not effectively reach the middle and lower clivus. Due to irregular shape of the middle turbinate bone, the MTF is often difficult to elevate, and resultant mucosa is often very thin and not always sufficient.

Regional vascular tissue flaps, such as the PCF and TPFF, are large, robust, and can reach the anterior cranial fossa, sella, parasellar, and clival defects. However, these flaps, especially the TPFF, require extra time and are technically more challenging. All flaps discussed here, local or regional, have a vascular pedicle, which is a significant advantage in promoting success of reconstruction and healing. We have also had the opportunity to use free flaps, usually the ALT flap but do so only when local and regional options are exhausted.

With careful planning and technical expertise, complications following EEA to the skull base can be greatly reduced with vascularized reconstructive options. Surgeons should, especially, be comfortable with the NSF as their primary reconstructive option; however, care and technical consideration need to be given to raising the flap with the same attention that is given to the approach and resection phases.

\section{Conflict of Interest}

Amin Kassam has the following disclosures:

(1) Synaptive Medical (consultant),

(2) KLS Martin (consultant), and

(3) Medtronic Medical (advisory board). Other authors have nothing to disclose.

\section{Acknowledgments}

We would like to thank Stryker Medical, Synaptive Medical, Nico Corporation, and Karl Storz for their support and assistance in the utilization of their technologies. We are also grateful for their support of the Neuroanatomy Laboratory.

\section{References}

1 Kassam AB, Prevedello DM, Carrau RL, et al. Endoscopic endonasal skull base surgery: analysis of complications in the authors' initial 800 patients. J Neurosurg 2011;114(06):1544-1568

2 Eloy JA, Kuperan AB, Choudhry OJ, Harirchian S, Liu JK. Efficacy of the pedicled nasoseptal flap without cerebrospinal fluid (CSF) diversion for repair of skull base defects: incidence of postoperative CSF leaks. Int Forum Allergy Rhinol 2012;2(05):397-401

3 Martín-Martín C, Martínez-Capoccioni G, Serramito-García R, Espinosa-Restrepo F. Surgical challenge: endoscopic repair of cerebrospinal fluid leak. BMC Res Notes 2012;5:459

4 Shin M, Kondo K, Saito N. Current status of endoscopic endonasal surgery for skull base meningiomas: review of the literature. Neurol Med Chir (Tokyo) 2015;55(09):735-743

5 Kassam A, Thomas AJ, Snyderman C, et al. Fully endoscopic expanded endonasal approach treating skull base lesions in pediatric patients. J Neurosurg 2007;106(2, Suppl)75-86

6 Patel MR, Stadler ME, Snyderman $\mathrm{CH}$, et al. How to choose? Endoscopic skull base reconstructive options and limitations. Skull Base 2010;20(06):397-404

7 Kassam AB, Thomas A, Carrau RL, et al. Endoscopic reconstruction of the cranial base using a pedicled nasoseptal flap. Neurosurgery 2008;63(01, Suppl 1):ONS44-ONS52, discussion ONS52-ONS53

8 Harvey RJ, Parmar P, Sacks R, Zanation AM. Endoscopic skull base reconstruction of large dural defects: a systematic review of published evidence. Laryngoscope 2012;122(02):452-459 
9 Hadar T, Ophir D, Yaniv E, Berger G. Inferior turbinate arterial supply: histologic analysis and clinical implications. J Otolaryngol 2005;34(01):46-50

10 Fortes FS, Carrau RL, Snyderman $\mathrm{CH}$, et al. The posterior pedicle inferior turbinate flap: a new vascularized flap for skull base reconstruction. Laryngoscope 2007;117(08):1329-1332

11 Harvey RJ, Sheahan PO, Schlosser RJ. Inferior turbinate pedicle flap for endoscopic skull base defect repair. Am J Rhinol Allergy 2009;23(05):522-526

12 Chiu T. A study of the maxillary and sphenopalatine arteries in the pterygopalatine fossa and at the sphenopalatine foramen. Rhinology 2009;47(03):264-270

13 Schwartzbauer HR, Shete M, Tami TA. Endoscopic anatomy of the sphenopalatine and posterior nasal arteries: implications for the endoscopic management of epistaxis. Am J Rhinol 2003;17(01):63-66

14 Yoshioka N, Rhoton AL Jr. Vascular anatomy of the anteriorly based pericranial flap. Neurosurgery 2005;57(1, Suppl)11-16; discussion 11-16

15 Patel MR, Shah RN, Snyderman CH, et al. Pericranial flap for endoscopic anterior skull-base reconstruction: clinical outcomes and radioanatomic analysis of preoperative planning. Neurosurgery 2010;66(03):506-512; discussion 512

16 Smith JE, Ducic Y. The versatile extended pericranial flap for closure of skull base defects. Otolaryngol Head Neck Surg 2004; 130(06):704-711
17 Perkins EL, Brandon BM, Sreenath SB, et al. Transfacial and craniofacial approaches for resection of sinonasal and ventral skull base malignancies. Otolaryngol Clin North Am 2017;50(02):287-300

18 David SK, Cheney ML. An anatomic study of the temporoparietal fascial flap. Arch Otolaryngol Head Neck Surg 1995;121(10): 1153-1156

19 Fortes FS, Carrau RL, Snyderman CH, et al. Transpterygoid transposition of a temporoparietal fascia flap: a new method for skull base reconstruction after endoscopic expanded endonasal approaches. Laryngoscope 2007;117(06):970-976

20 Eloy JA, Patel AA, Shukla PA, Choudhry OJ, Liu JK. Early harvesting of the vascularized pedicled nasoseptal flap during endoscopic skull base surgery. Am J Otolaryngol 2013;34(03):188-194

21 Liu JK, Schmidt RF, Choudhry OJ, Shukla PA, Eloy JA. Surgical nuances for nasoseptal flap reconstruction of cranial base defects with high-flow cerebrospinal fluid leaks after endoscopic skull base surgery. Neurosurg Focus 2012;32(06):E7

22 Hadad G, Bassagasteguy L, Carrau RL, et al. A novel reconstructive technique after endoscopic expanded endonasal approaches: vascular pedicle nasoseptal flap. Laryngoscope 2006;116(10): 1882-1886

23 Pinheiro-Neto CD, Prevedello DM, Carrau RL, et al. Improving the design of the pedicled nasoseptal flap for skull base reconstruction: a radioanatomic study. Laryngoscope 2007;117(09): 1560-1569 